\title{
Investigation of transmembrane proteins using a computational
} approach

\author{
Jack Y Yang ${ }^{1}$, Mary Qu Yang'2, Keith A Dunker ${ }^{3}$, Youping Deng*4 and \\ Xudong Huang*1
}

\begin{abstract}
Address: ${ }^{1}$ Department of Radiology, Brigham and Women's Hospital and Harvard Medical School, Boston, MA 02115, USA, ${ }^{2}$ National Human Genome Research Institute, National Institutes of Health, Bethesda, MD 20892, USA, ${ }^{3}$ Center for Computational Biology and Bioinformatics, Indiana University Schools of Medicine and Informatics, 410 W. 10th Street, Indianapolis, IN 46202, USA and ${ }^{4}$ Department of Biological Sciences, University of Southern Mississippi, Hattiesburg, 39406, USA
\end{abstract}

Email: Jack Y Yang - jyang@bwh.harvard.edu; Mary Qu Yang - yangma@mail.nih.gov; Keith A Dunker - kedunker@iupui.edu; Youping Deng* - youping.deng@usm.edu; Xudong Huang* - xhuang3@partners.org

* Corresponding authors

from The 2007 International Conference on Bioinformatics \& Computational Biology (BIOCOMP'07)

Las Vegas, NV, USA. 25-28 June 2007

Published: 20 March 2008

BMC Genomics 2008, 9(Suppl I):S7 doi:10.1186/147I-2164-9-SI-S7

This article is available from: http://www.biomedcentral.com/I47I-2I64/9/SI/S7

(c) 2008 Yang et al.; licensee BioMed Central Ltd.

This is an open access article distributed under the terms of the Creative Commons Attribution License (http://creativecommons.org/licenses/by/2.0), which permits unrestricted use, distribution, and reproduction in any medium, provided the original work is properly cited.

\begin{abstract}
Background: An important subfamily of membrane proteins are the transmembrane $\alpha$-helical proteins, in which the membrane-spanning regions are made up of $\alpha$-helices. Given the obvious biological and medical significance of these proteins, it is of tremendous practical importance to identify the location of transmembrane segments. The difficulty of inferring the secondary or tertiary structure of transmembrane proteins using experimental techniques has led to a surge of interest in applying techniques from machine learning and bioinformatics to infer secondary structure from primary structure in these proteins. We are therefore interested in determining which physicochemical properties are most useful for discriminating transmembrane segments from non-transmembrane segments in transmembrane proteins, and for discriminating intrinsically unstructured segments from intrinsically structured segments in transmembrane proteins, and in using the results of these investigations to develop classifiers to identify transmembrane segments in transmembrane proteins.
\end{abstract}

Results: We determined that the most useful properties for discriminating transmembrane segments from nontransmembrane segments and for discriminating intrinsically unstructured segments from intrinsically structured segments in transmembrane proteins were hydropathy, polarity, and flexibility, and used the results of this analysis to construct classifiers to discriminate transmembrane segments from non-transmembrane segments using four classification techniques: two variants of the Self-Organizing Global Ranking algorithm, a decision tree algorithm, and a support vector machine algorithm. All four techniques exhibited good performance, with out-of-sample accuracies of approximately $75 \%$.

Conclusions: Several interesting observations emerged from our study: intrinsically unstructured segments and transmembrane segments tend to have opposite properties; transmembrane proteins appear to be much richer in intrinsically unstructured segments than other proteins; and, in approximately $70 \%$ of transmembrane proteins that contain intrinsically unstructured segments, the intrinsically unstructured segments are close to transmembrane segments. 


\section{Background}

Membrane proteins account for roughly one third of all proteins and play a crucial role in processes such as cellto-cell signaling, transport of ions across membranes, and energy metabolism [1-3], and are a prime target for therapeutic drugs [2,4-6]. One important subfamily of membrane proteins are the transmembrane proteins, of which there are two main types:

- $\alpha$-helical proteins, in which the membrane-spanning regions are made up of $\alpha$-helices, and

- $\beta$-barrel proteins, in which the membrane-spanning regions are made up of $\beta$-strands.

$\beta$-barrel proteins are found mainly in the outer membrane of gram-negative bacteria, and possibly in eukaryotic organelles such as mitochondria, whereas $\alpha$-helical proteins are found in eukaryotes and the inner membranes of bacteria [7].

Given the obvious biological and medical significance of transmembrane proteins, it is of tremendous practical importance to identify the location of transmembrane segments. There are difficulties with obtaining the three dimensional structure of membrane proteins using experimental techniques:

- Membrane proteins have both a hydrophilic part and a hydrophobic part, and hence are not entirely soluble in either aqueous or organic solvents; this makes them difficult to crystallize, and hence difficult to analyze using Xray crystallography, which requires crystallization of the sample.

- Membrane proteins tend to denature upon removal from the membrane, making their three-dimensional structure difficult to analyze.

The difficulty of inferring the secondary or tertiary structure of transmembrane proteins using experimental techniques has led to a surge of interest in applying techniques from machine learning and bioinformatics to infer secondary structure from primary structure in these proteins. These include discriminant analysis [8], decision trees [9], neural networks [10-13], support vector machines [14$18]$, and hidden Markov models [19,20].

Another interesting class of proteins are the intrinsically unstructured proteins, proteins that need not be folded into a particular configuration to carry out their function, existing instead as dynamic ensembles in their native state [21-24]. Intrinsically unstructured proteins have been associated with a wide range of functions including molecular recognition, molecular assembly/disassembly and protein modification $[21,22,25]$.

We are interested in investigating the physicochemical properties of various classes of protein segments. In particular, we are interested in determining which properties are useful for discriminating transmembrane segments from non-transmembrane segments in transmembrane proteins, and for discriminating intrinsically unstructured segments from intrinsically structured segments in transmembrane proteins. We are further interested in any similarities or differences in physicochemical properties across these four classes of segments. We will then apply the results of this analysis to construct classifiers to discriminate transmembrane from non-transmembrane segments in transmembrane proteins.

\section{Results and discussion \\ Physicochemical properties}

We are interested in determining which physicochemical properties are most useful for discriminating transmembrane segments from non-transmembrane segments in transmembrane proteins, and for discriminating intrinsically unstructured segments from intrinsically structured segments in transmembrane proteins. We are further interested in any similarities or differences in physicochemical properties across these four classes of segments.

Certain properties, such as hydropathy and polarity, can be measured in different ways; this results in different scales. We are also interested in determining which scales are the most effective in discriminating transmembrane segments from non-transmembrane segments, and in discriminating intrinsically unstructured from intrinsically structured segments in transmembrane proteins.

Our interest is in properties that can be easily computed given only a sequence of amino acids; we therefore considered properties that depend only on the type of each amino acid in a sequence, including:

- Hydropathy, a measure of the relative hydrophobicity of an amino acid. There are four hydropathy scales in common use - the Kyte-Doolittle [26], Eisenberg-SchwarzKomaromy-Wall [27], Engelman-Steitz-Goldman [28], and Liu-Deber [29] scales.

- Polarity, a measure of how charge is distributed over an amino acid, affects how amino acids interact, and helps to determine protein structure. There are two polarity scales in common use-the Grantham [30] and the Zimmerman-Eleizer-Simha [31] scales.

- Flexibility, a measure of the amount to which an amino acid residue contributes to the flexibility of a protein. 
- Polarizability, a measure of the extent to which positive and negative charge can be separated in the presence of an applied electric field.

- van der Waals volume, a measure of the volume occupied by an amino acid.

- Bulkiness, a measure of the volume occupied by an amino acid, is correlated with hydrophobicity [32].

- Electronic effects, a measure that takes into account steric factors, inductive effects, resonance effects, and field effects [33].

- Helicity, the propensity of an amino acid to contribute to the formation of helical structures in proteins [34].

Given a sequence of amino acids, the "pointwise" property value associated to a particular position in the sequence depends only on which of the 20 amino acids occurs at that position. To increase the robustness of our results, we work with average property values instead of pointwise property values. The average of a given property associated to a particular amino acid $A$ in the sequence is the average of the pointwise property values associated to the amino acids contained in a window of length $L$ centered at $A$. The effectiveness of each property at discriminating transmembrane from non-transmembrane segments and intrinsically unstructured from intrinsically structured segments was assessed based on two criteria:

(1) For a given property $X$, the degree to which the classconditional distributions for the two classes overlap, that is, the degree to which $p_{X}(x \mid$ class 1$)$ and $p_{X}$ (x|class 2$)$ overlap. The less these two probability distributions overlap, the more easily the two classes can be separated. Knowledge of these probability distributions forms the basis for a Bayesian classifier, which classifies an instance having a value $x$ for property $X$ to "class 1 " if and only if

$$
\frac{p x(x \mid \operatorname{class} 1)}{p x(x \mid \operatorname{class} 2)}>\frac{P\{\operatorname{class} 2\}}{P\{\operatorname{class} 1\}}
$$

where $P\{$ class 1$\}$ is the probability of observing a class 1 instance and $P\{$ class 2$\}$ is the probability of observing a class 2 instance. The class-conditional probability distributions for the above properties are plotted in Figures $1,2,3$.

(2) The Overlap Ratio, defined in the Methods section, is a numerical measure of the overlap between the conditional probabilities $\mathrm{P}\{$ class $1 \mid X=x\}$ and $\mathrm{P}\{$ class $2 \mid X=x\}$. The smaller the Overlap Ratio, the more easily the two classes can be discriminated.
The Overlap Ratios for discriminating transmembrane from non-transmembrane segments are shown in Table 1, while the Overlap Ratios for discriminating intrinsically unstructured from intrinsically structured segments are shown in Table 2. It turns out that the discriminating power of a given property depends on the length $L$ of the window over which property values are averaged; Overlap Ratios are given in Tables 1 and 2 for all odd values of the window length $L$ between 9 and 31 .

Our conclusions were as follows:

- Whereas all four hydropathy scales can be used for discriminating transmembrane segments for non-transmembrane segments in transmembrane proteins, the LiuDeber scale is the best scale for this task.

- Whereas all four hydropathy scales can be used for discriminating intrinsically unstructured segments from intrinsically structured segments in transmembrane proteins, the Eisenberg-Schwarz-Komaromy-Wall scale is the best scale for this task.

- Whereas both polarity scales can be used for discriminating transmembrane from non-transmembrane segments and for discriminating intrinsically unstructured from intrinsically structured segments in transmembrane proteins, the Grantham scale is slightly better for these tasks.

- For both classification problems (discriminating transmembrane from non-transmembrane segments and discriminating intrinsically unstructured from intrinsically structured segments), flexibility provided some degree of discriminating power, and bulkiness provided still less; neither property was as effective as hydropathy or polarity at discriminating between the two classes.

- For both classification problems, polarizability, van der Waals volume, electronic effects, and helicity did not discriminate well between the two classes.

\section{Transmembrane segment classifiers}

We tested four classification techniques on the problem of discriminating transmembrane segments from non-transmembrane segments in transmembrane proteins:

- C4.5 [35], a decision tree algorithm.

- SVM ${ }^{\text {light }}$ version 6.01 (linear kernel function) [36], a support vector machine algorithm.

- Two variants of the Self-Organizing Global Ranking (SOGR) algorithm [37], SOGR-I [38,39] and SOGR-IB $[38,39]$, which are described in detail in the Methods sec- 
(a) TM vs. Non-TM

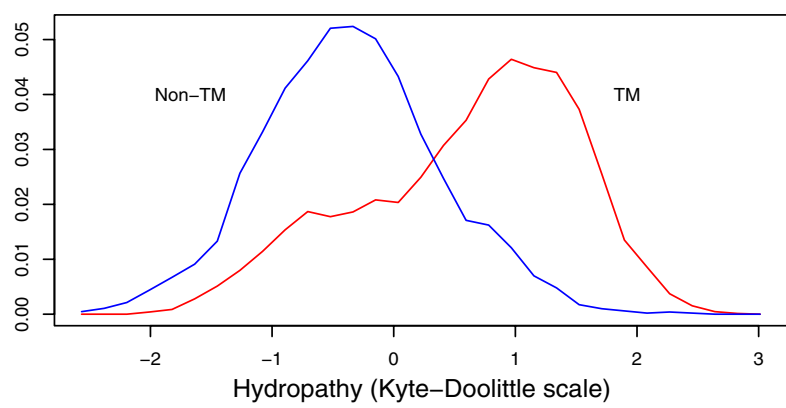

(c) TM vs. Non-TM

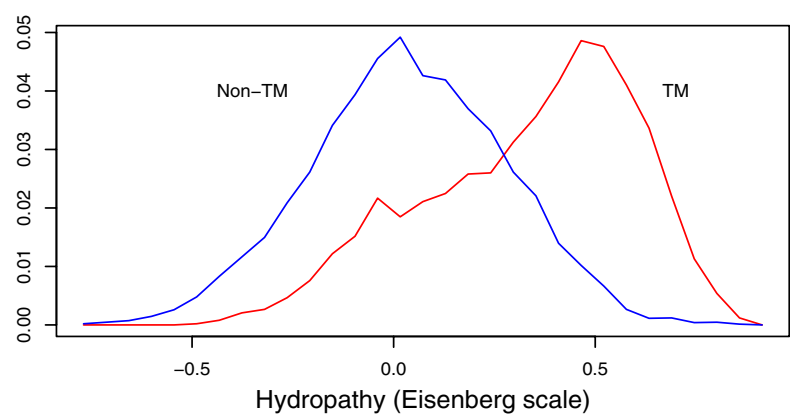

(e) TM vs. Non-TM

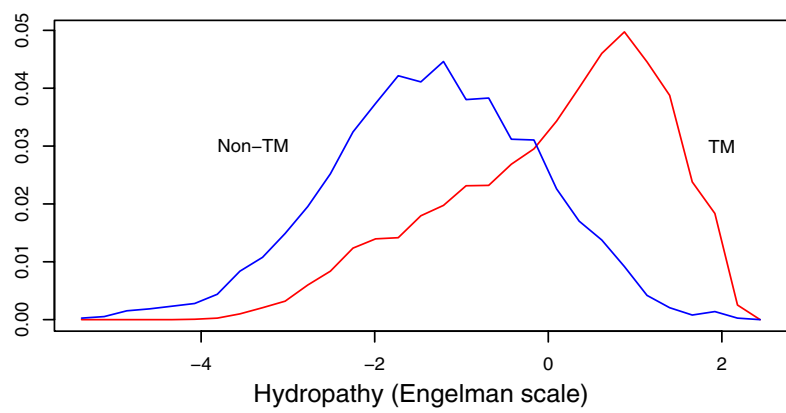

(g) TM vs. Non-TM

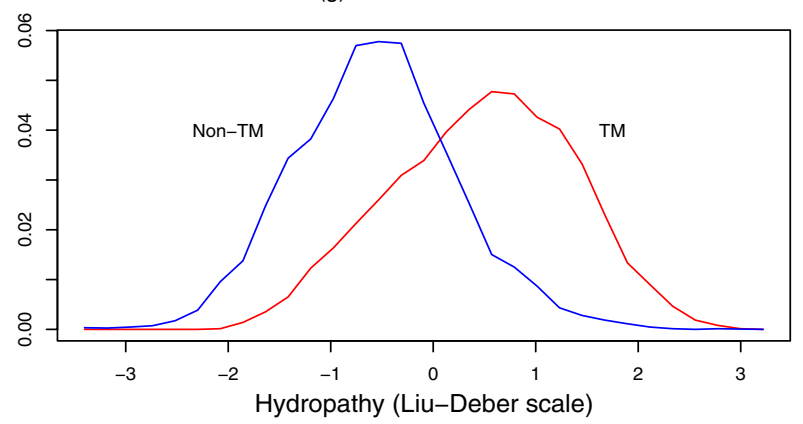

(b) IU vs. Non-IU

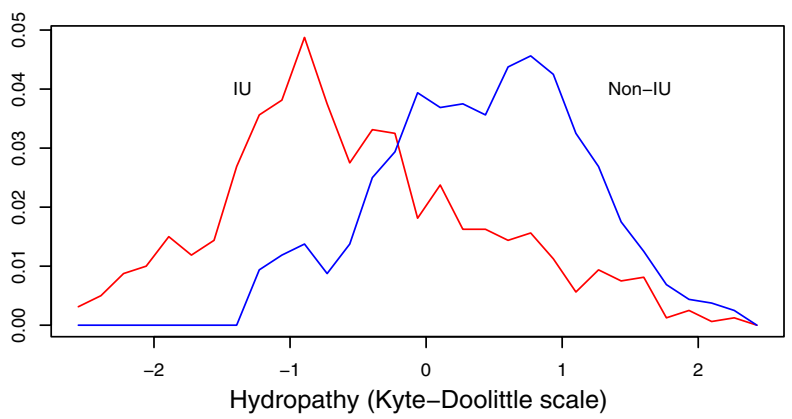

(d) IU vs. Non-IU

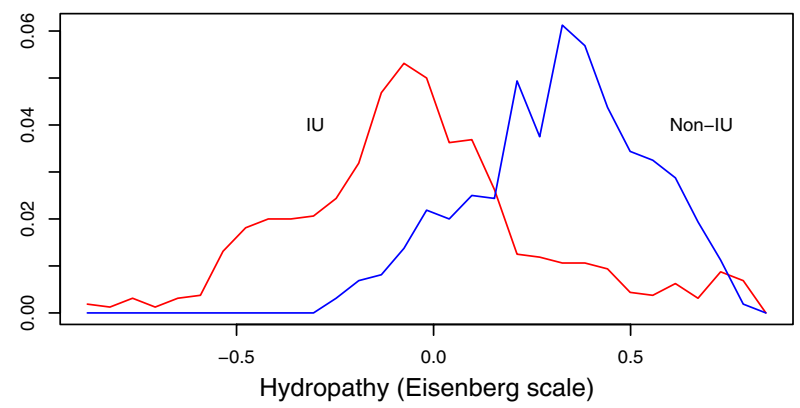

(f) IU vs. Non-IU

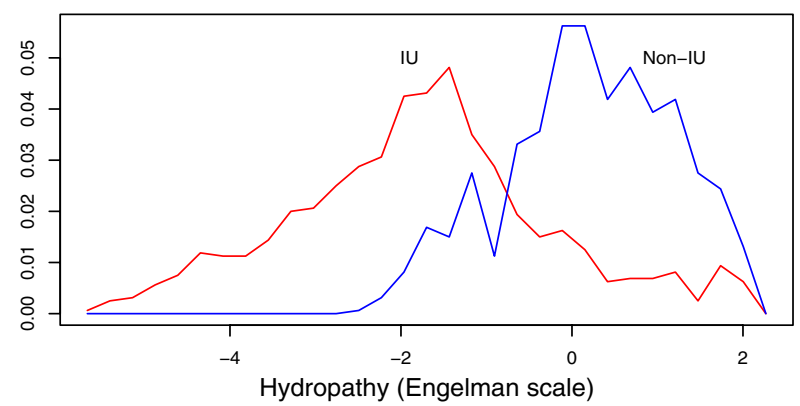

(h) IU vs. Non-IU

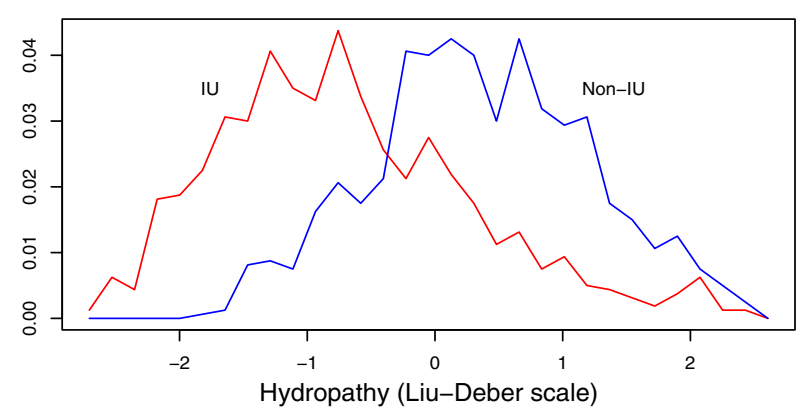

Figure I

Conditional probability distributions $p(x \mid \mathrm{TM}), p(x \mid$ Non-TM) (on the left), and $p(x \mid \mathrm{IU}), p(x \mid$ Non-IU) (on the right), where $x$ is hydropathy, as determined by the Kyte-Doolittle, Eisenberg-Schwarz- Komaromy-Wall, Engelman-Steitz-Goldman, and LiuDeber scales. TM = transmembrane, IU = intrinsi-cally unstructured. The plots on the left were reproduced with permission from [38]. 
(a) TM vs. Non-TM

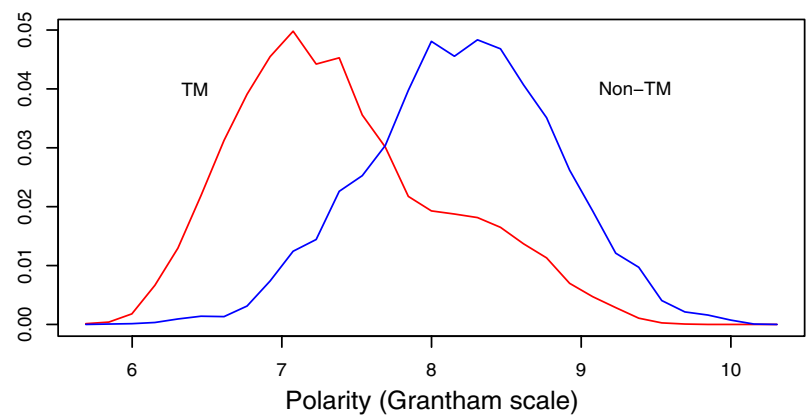

(c) TM vs. Non-TM

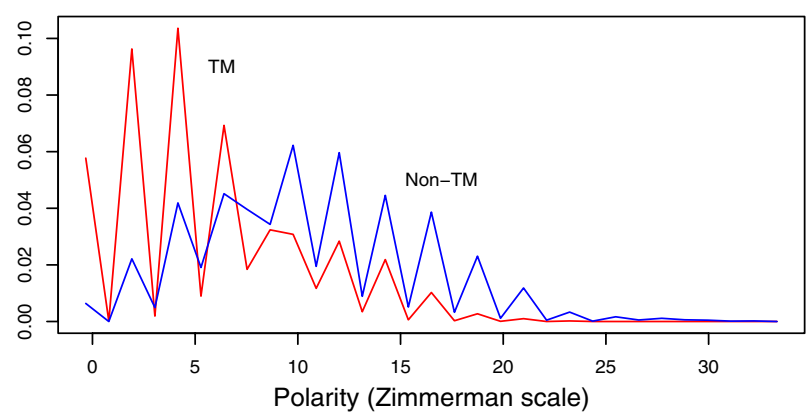

(e) TM vs. Non-TM

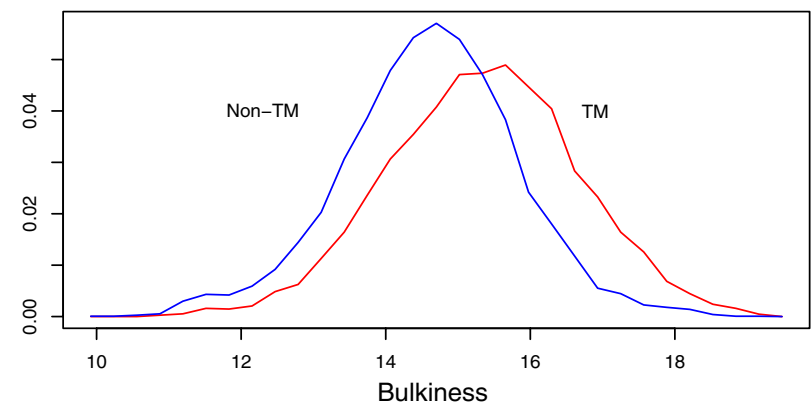

(g) TM vs. Non-TM

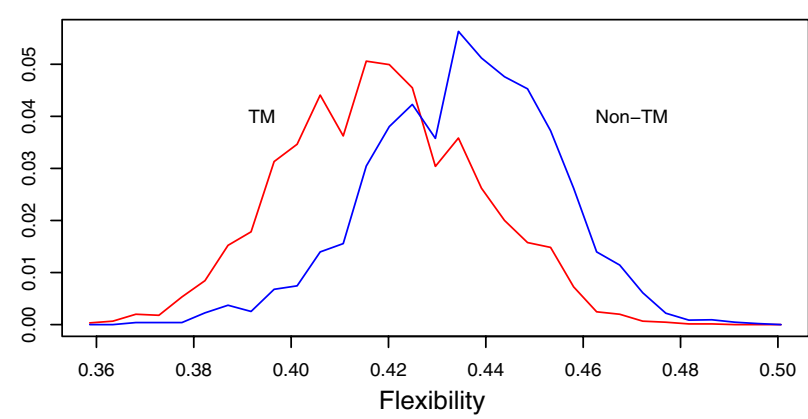

(b) IU vs. Non-IU

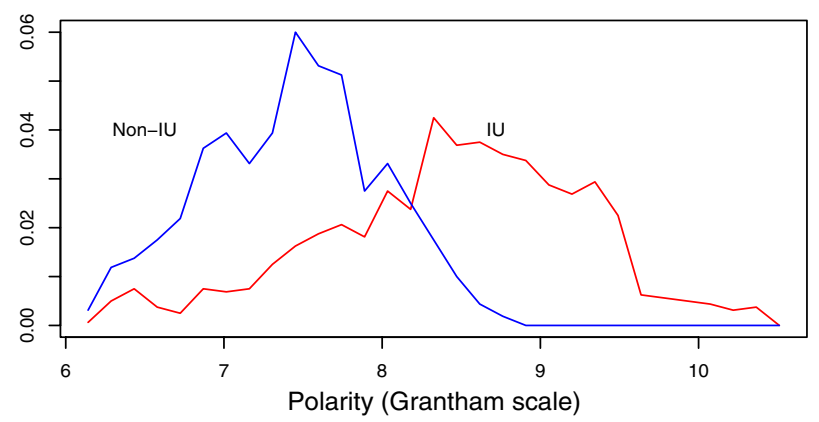

(d) IU vs. Non-IU

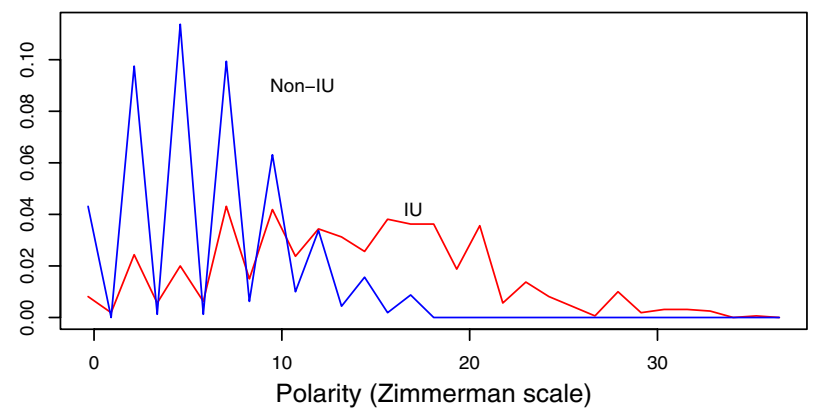

(f) IU vs. Non-IU

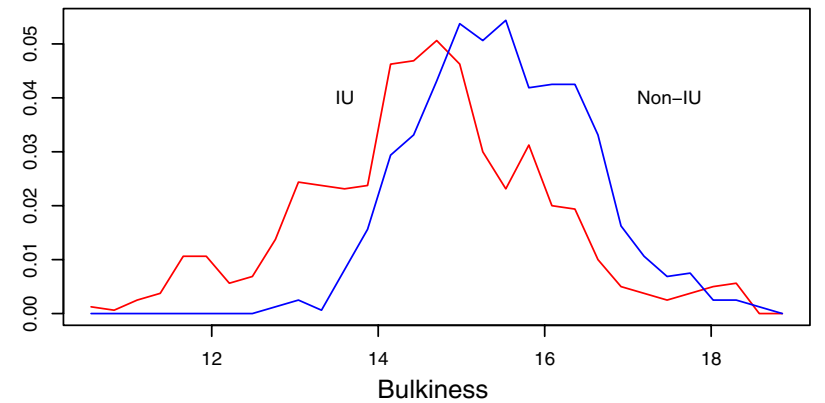

(h) IU vs. Non-IU

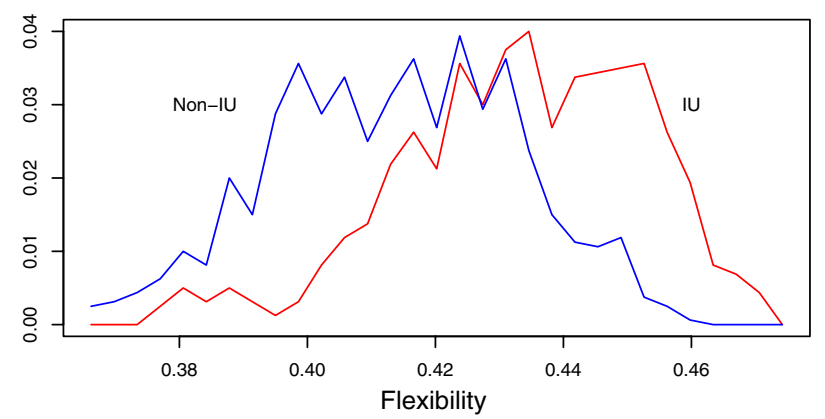

Figure 2

Conditional probability distributions $p(x \mid$ TM), $p(x \mid$ Non-TM) (on the left), and $p(x \mid I U), p(x \mid$ Non-IU) (on the right), where $x$ is, from top to bottom, polarity, as determined by the Grantham and Zimmerman-Eleizer-Simha scales, bulkiness, and flexibility. $\mathrm{TM}=$ transmembrane, IU = intrinsically unstructured. The plots on the left were reproduced with permission from [38]. 
(a) TM vs. Non-TM

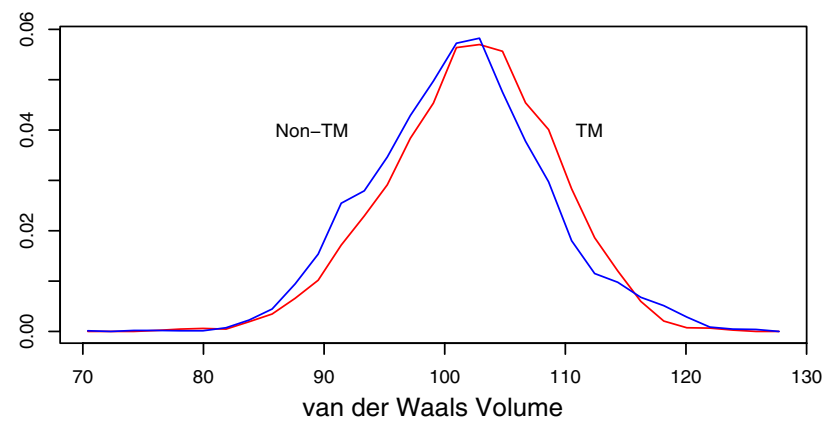

(c) TM vs. Non-TM

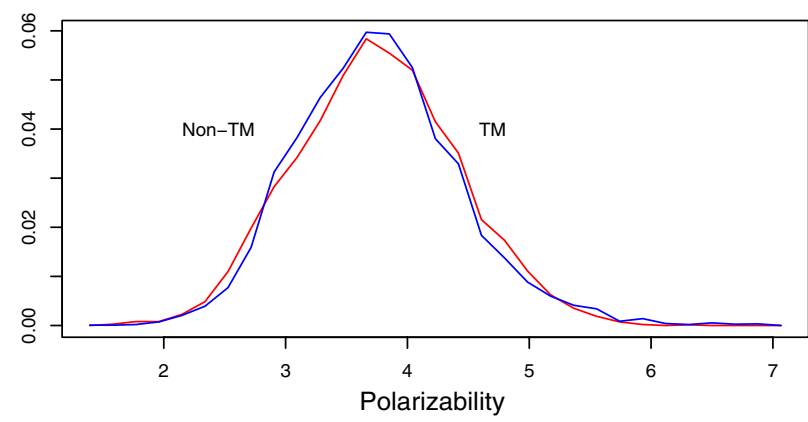

(e) TM vs. Non-TM

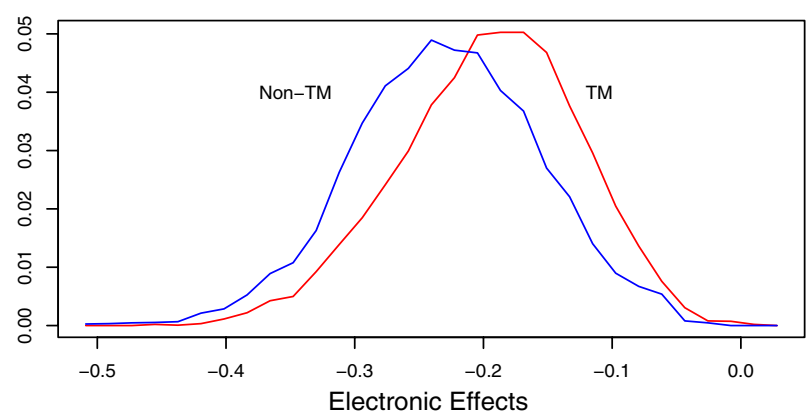

(g) TM vs. Non-TM

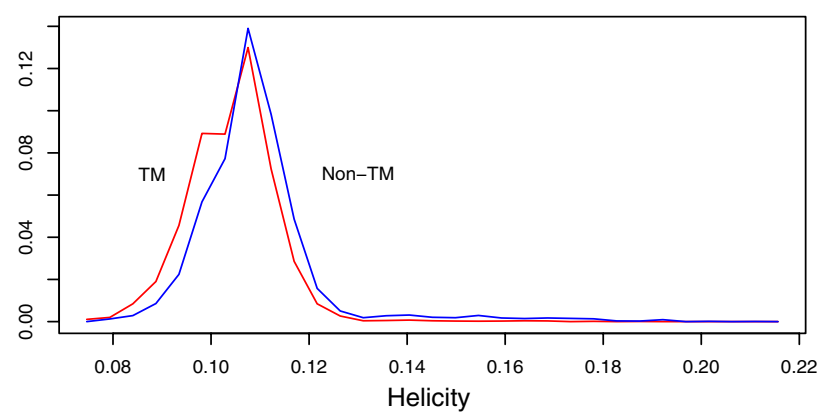

(b) IU vs. Non-IU

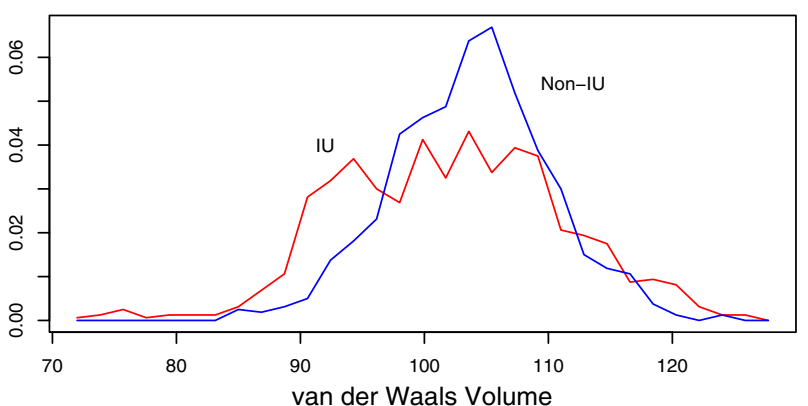

(d) IU vs. Non-IU

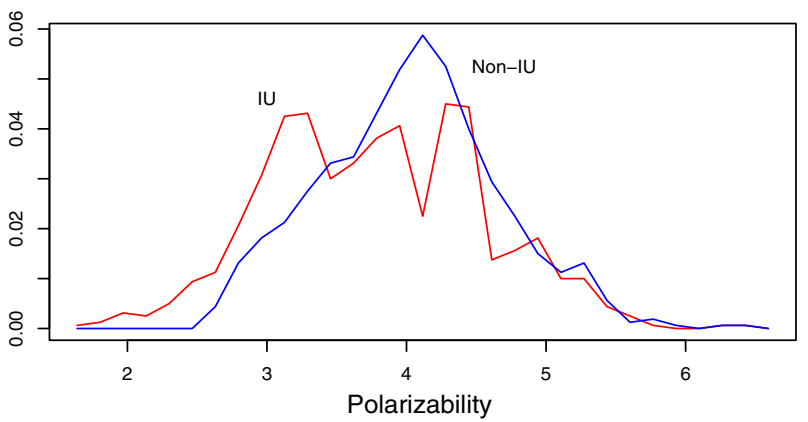

(f) IU vs. Non-IU

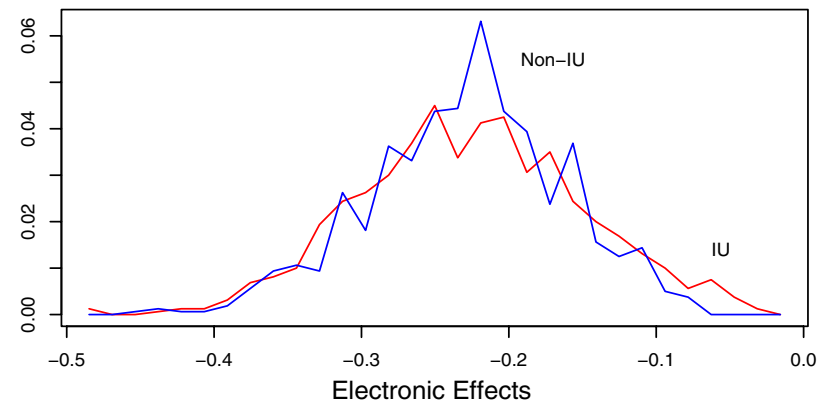

(h) IU vs. Non-IU

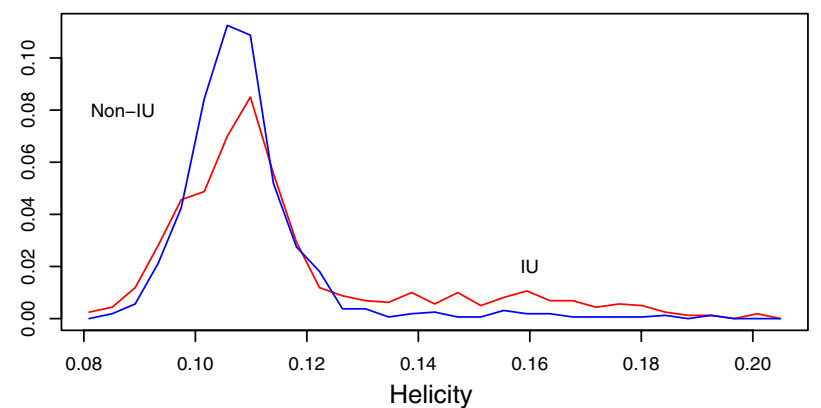

\section{Figure 3}

Conditional probability distributions $p(x \mid \mathrm{TM}), p(x \mid$ Non-TM) (on the left), and $p(x \mid \mathrm{IU}), p(x \mid$ Non-IU) (on the right), where $x$ is, from top to bottom, van der Waals volume, polarizability, elec-tronic effects, and helicity. TM $=$ transmembrane, IU $=$ intrinsically unstructured. The plots on the left were reproduced with permission from [38]. 
Table I: Overlap Ratios for discriminating transmembrane segments from non-transmembrane segments in membrane proteins as a function of window length (W.L.).

\begin{tabular}{llllllllll}
\hline W.L. & $H_{K D}$ & $H_{E i}$ & $H_{E n}$ & $H_{L D}$ & $\boldsymbol{P}_{\boldsymbol{G}}$ & $\boldsymbol{P}_{\boldsymbol{Z}}$ & Bulk. & Flex. & Elec. \\
\hline 31 & 0.249 & 0.221 & 0.260 & 0.198 & 0.249 & 0.211 & 0.423 & 0.294 & 0.504 \\
29 & 0.232 & 0.197 & 0.241 & 0.183 & 0.223 & 0.223 & 0.397 & 0.278 & 0.499 \\
27 & 0.231 & 0.203 & 0.213 & 0.194 & 0.232 & 0.232 & 0.412 & 0.266 & 0.462 \\
25 & 0.238 & 0.198 & 0.227 & 0.178 & 0.215 & 0.269 & 0.393 & 0.269 & 0.411 \\
23 & 0.217 & 0.204 & 0.219 & 0.177 & 0.208 & 0.233 & 0.385 & 0.258 & 0.434 \\
21 & 0.209 & 0.204 & 0.215 & 0.166 & 0.216 & 0.197 & 0.370 & 0.252 & 0.379 \\
19 & 0.214 & 0.222 & 0.220 & 0.199 & 0.224 & 0.235 & 0.415 & 0.259 & 0.389 \\
17 & 0.201 & 0.252 & 0.218 & 0.199 & 0.219 & 0.206 & 0.393 & 0.259 & 0.442 \\
15 & 0.191 & 0.195 & 0.201 & 0.214 & 0.224 & 0.193 & 0.356 & 0.283 & 0.456 \\
13 & 0.216 & 0.203 & 0.217 & 0.178 & 0.203 & 0.189 & 0.325 & 0.283 & 0.500 \\
11 & 0.210 & 0.199 & 0.228 & 0.185 & 0.204 & 0.168 & 0.346 & 0.277 & 0.493 \\
9 & 0.231 & 0.205 & 0.222 & 0.200 & 0.232 & 0.280 & 0.396 & 0.299 & 0.562 \\
\hline
\end{tabular}

Here $H_{K D}, H_{E \dot{p}} H_{E n}, H_{L D}$ indicate the Kyte-Doolittle, Eisenberg-Schwarz-Komaromy-Wall, Engelman-Steitz-Goldman, and Liu-Deber hydropathy scales, respectively, $P_{G}, P_{z}$ indicate the Grantham and Zimmerman-Eliezer-Simha polarity scales, respectively, Bulk. $=$ bulkiness, Flex. $=$ flexibility, and Elec. = electronic effects.

Reproduced with permission from [38]

tion. These algorithms depend on a number of parameters: the length $L$ of the window used to extract features, the number of neurons $m$, the learning rate $\eta_{t}$, and the neighborhood size $R$. The performance of these algorithms depends on the choice of these parameters: For example, the performance of the SOGR-I algorithm as a function of the length of the window used to extract features is shown in Figure 4. Based on a series of experiments, we settled on feature window length $L$ of 10 , a network size $m$ of 16 neurons, a fixed learning rate $\eta_{t}$ of .05 , and a neighborhood size $R$ of 2 . Since the length of the window used to extract features was chosen to maximize the performance of the SOGR-I algorithm, the results will be slightly biased in favor of the SOGR-I and SOGR-IB algorithms.

Designing a classifier also involves selecting the features that are most useful for the problem of interest. Based on our investigations of physicochemical properties, we based the classification on three features:

- Hydropathy (Liu-Deber scale)

- Polarity (Grantham scale)

- Flexibility

Table 2: Overlap Ratios for discriminating intrinsically unstructured segments from intrinsically structured segments in membrane proteins as a function of window length (W.L.).

\begin{tabular}{lllllllll}
\hline W.L. & $H_{K D}$ & $H_{E i}$ & $H_{E n}$ & $H_{L D}$ & $P_{G}$ & $P_{Z}$ & Bulk. & Flex. \\
\hline 31 & 0.318 & 0.163 & 0.170 & 0.243 & 0.220 & 0.134 & 0.349 & 0.227 \\
29 & 0.221 & 0.229 & 0.167 & 0.249 & 0.138 & 0.161 & 0.351 & 0.238 \\
27 & 0.222 & 0.150 & 0.164 & 0.230 & 0.170 & 0.142 & 0.221 & 0.263 \\
25 & 0.216 & 0.234 & 0.162 & 0.241 & 0.175 & 0.142 & 0.364 & 0.272 \\
23 & 0.253 & 0.143 & 0.160 & 0.253 & 0.163 & 0.157 & 0.238 & 0.254 \\
21 & 0.182 & 0.139 & 0.144 & 0.267 & 0.176 & 0.159 & 0.323 & 0.271 \\
19 & 0.285 & 0.142 & 0.149 & 0.257 & 0.172 & 0.251 & 0.337 & 0.291 \\
17 & 0.290 & 0.199 & 0.148 & 0.266 & 0.183 & 0.307 & 0.353 & 0.279 \\
15 & 0.320 & 0.170 & 0.155 & 0.274 & 0.182 & 0.183 & 0.338 & 0.361 \\
13 & 0.264 & 0.180 & 0.165 & 0.284 & 0.194 & 0.254 & 0.358 & 0.340 \\
11 & 0.310 & 0.228 & 0.195 & 0.281 & 0.220 & 0.446 & 0.345 & 0.358 \\
9 & 0.372 & 0.230 & 0.226 & 0.325 & 0.269 & 0.251 & 0.416 & 0.401
\end{tabular}

Here $H_{K D}, H_{E,} H_{E n}, H_{L D}$ indicate the Kyte-Doolittle, Eisenberg-Schwarz-Komaromy-Wall, Engelman-Steitz-Goldman, and Liu-Deber hydropathy scales, respectively, $P_{G}, P_{Z}$ indicate the Grantham and Zimmerman-Eliezer-Simha polarity scales, respectively, Bulk. $=$ bulkiness, and Flex. $=$ flexibility.

Reproduced with permission from [38] 


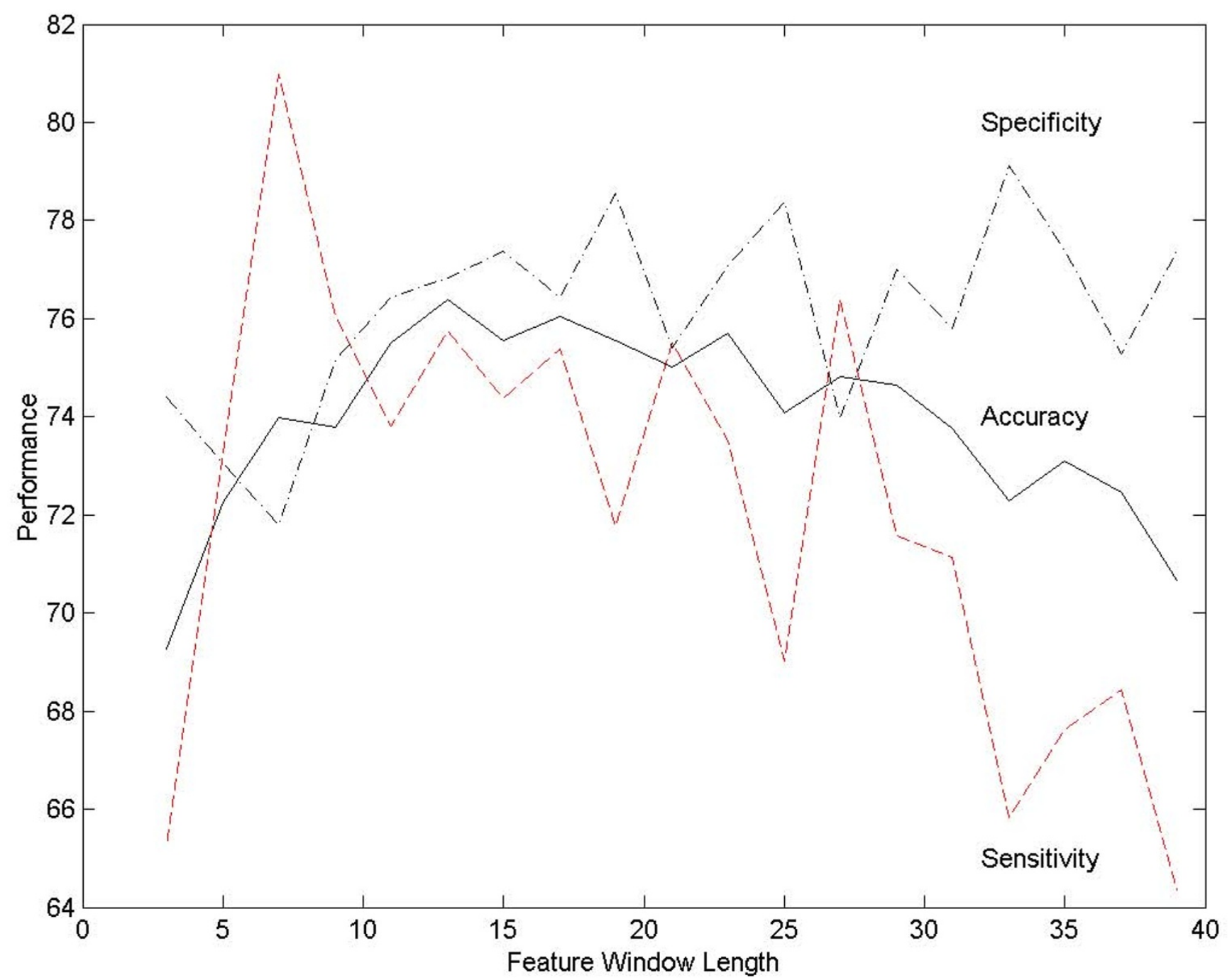

Figure 4

Performance of the SOGR-I classifier as a function of the length of the window used to extract features, based on threefold cross-validation (fixed learning rate $\eta_{t}=.05$, neighborhood size $R=2$, number of neurons $=16$ ). Reproduced with permission from [38].

The performance of the above four classification techniques under ten-fold cross-validation when hydropathy (Liu-Deber scale), polarity (Grantham scale), and flexibility are used as features is shown in Table 3, while the performance when only polarity (Grantham scale) and flexibility are used as features is shown in Table 4. It is interesting that performance drops only slightly when two features are used instead of three. All four classifiers exhibited good performance, with out-of-sample accuracies of approximately $75 \%$. While this may seem low, the substantial overlap of the transmembrane and non-transmembrane classes seen in Figures 1,2,3 makes this a nontrivial classification problem. Filtering strategies can be used to improve the performance of these classifiers [38,39].

\section{Conclusions}

We determined that the most useful properties for discriminating transmembrane segments from non-transmembrane segments and for discriminating intrinsically unstructured segments from intrinsically structured segments in transmembrane proteins were hydropathy, polarity, and flexibility, and based on these properties, constructed a number of classifiers to identify transmembrane segments with an out-of-sample accuracy of approximately $75 \%$. Several interesting observations emerged from our study: 
Table 3: Accuracy of discriminating transmembrane segments from non-transmembrane segments in trans-membrane proteins using the SOGR-I and SOGR-IB classifiers, a decision tree classifier (C4.5), and a support vector machine classifier (SVMlight version 6.0I), based on ten-fold cross-validation. Three features were used, namely hydropathy (Liu-Deber scale), polarity (Grantham scale), and flexibility.

\begin{tabular}{llllll}
\hline Fold & SOGR-I & SOGR-IB & $\begin{array}{l}\text { C4.5 } \\
\text { Before Pruning }\end{array}$ & After Pruning & SVM \\
\hline 1 & 72.2311 & 72.2311 & 72.4960 & 72.5490 & 72.9730 \\
2 & 69.0476 & 67.1733 & 67.8318 & 67.6798 & 67.3252 \\
3 & 77.1277 & 76.9149 & 77.5532 & 77.6596 & 83.7660 \\
4 & 81.8913 & 84.5875 & 83.7827 & 83.7827 & 78.4608 \\
5 & 79.3146 & 78.4889 & 78.3237 & 78.4476 & 82.0780 \\
6 & 81.4600 & 83.6230 & 82.8119 & 75.1595 & 76.3100 \\
7 & 75.9410 & 76.8266 & 75.6458 & 79.9891 & 79.2783 \\
8 & 78.3488 & 79.2783 & 79.8797 & 64.5543 & 64.7632 \\
9 & 64.1365 & 65.0418 & 64.5543 & 67.0111 & 75.6753 \\
10 & 67.2325 & 65.6089 & 66.8635 & 6.8 & 75.0 \\
Mean & 74.7 & 75.0 & 75.0 & 6.8 & 6.5 \\
Std. dev. & 6.2 & 7.2 & & & \\
\hline
\end{tabular}

Reproduced with permission from [38]

- Intrinsically unstructured segments and transmembrane segments tend to have opposite properties, as summarized in Table 5. For example, unstructured segments tended to have a low hydropathy value, whereas transmembrane segments tended to have a high hydropathy value. These results are in agreement with previous work that found that transmembrane segments tend to be more hydrophobic than non-transmembrane segments, due to the fact that transmembrane $\alpha$-helices require a stretch of 12-35 hydrophobic amino acids to span the hydrophobic region inside the membrane [26].

- Transmembrane proteins appear to be much richer in intrinsically unstructured segments than other proteins; about $70 \%$ of transmembrane proteins contain intrinsically unstructured regions, as compared to about 35\% of other proteins.

- In approximately $70 \%$ of transmembrane proteins that contain intrinsically unstructured segments, the intrinsically unstructured segments are close to transmembrane segments.

These observations may provide insight into the structural and functional roles that intrinsically unstructured segments play in membrane proteins, and may also aid in the identification of intrinsically unstructured and transmembrane segments from primary protein structure.

Table 4: Accuracy of discriminating transmembrane segments from non-transmembrane segments in trans-membrane proteins using the SOGR-I and SOGR-IB classifiers, a decision tree classifier (C4.5), and a support vector machine classifier (SVMlight version 6.0I), based on ten-fold cross-validation. Two features were used, namely polarity (Grantham scale) and flexibility.

\begin{tabular}{llllll}
\hline Fold & SOGR-I & SOGR-IB & $\begin{array}{l}\text { C4.5 } \\
\text { Before Pruning }\end{array}$ & After Pruning & SVM \\
\hline 1 & 71.7541 & 72.0721 & 72.3900 & 72.6020 & 72.6550 \\
2 & 65.1469 & 65.8561 & 66.1601 & 66.1601 & 67.0719 \\
3 & 77.1277 & 78.4043 & 76.3830 & 77.5532 & 77.4468 \\
4 & 83.0986 & 85.0302 & 83.7827 & 83.7827 & 83.0181 \\
5 & 77.2502 & 77.6631 & 76.4244 & 76.4244 & 82.1082 \\
6 & 81.9235 & 83.2368 & 82.8505 & 82.8119 & 75.8672 \\
7 & 75.5720 & 76.6052 & 75.7934 & 79.4970 & 79.410 \\
8 & 79.4423 & 79.4423 & 79.7704 & 64.2061 & 64.4150 \\
9 & 64.1365 & 64.3454 & 64.2061 & 67.0849 & 67.0849 \\
10 & 67.4539 & 67.5277 & 67.0849 & 74.6 & 74.8 \\
Mean & 74.3 & 75.0 & 74.5 & 6.9 & 6.7 \\
Std. dev. & 6.8 & 7.2 & 6.9 & & \\
\hline
\end{tabular}

Reproduced with permission from [38] 
Table 5: Tendencies of various properties for tranmembrane (TM) and intrinsically unstructured (IU) segments.

\begin{tabular}{|c|c|c|}
\hline & Segment & Type \\
\hline Property & $\mathrm{TM}$ & IU \\
\hline Hydropathy & High & Low \\
\hline Polarity & Low & High \\
\hline Bulkiness & High & Low \\
\hline Flexibility & Low & High \\
\hline Electronic effects & High & Low \\
\hline
\end{tabular}

Reproduced with permission from [38]

\section{Methods}

\section{Physicochemical properties}

The Overlap Ratio, a quantitative measure of how well two classes (referred to generically as "class 1 " and "class $2^{\prime \prime}$ ) can be discriminated based on a property $X$, was calculated as follows.

1. We construct a graph such that:

(a) The horizontal axis corresponds to the property $\mathrm{X}$. We divide this axis into bins.

(b) The $y$-value associated with the bin corresponding to $\mathrm{X}$ values between $x$ and $x+\in$ is the fraction of all instances in the training set that belong to class 1 and have a value for the feature $\mathrm{X}$ in the range $[x, x+\in)$, where $\in>0$ is small.

The graph represents an approximation to the function $\mathrm{P}\{$ class $1 \mid X=x\}$. We define the complementary function $\mathrm{P}\{$ class $2 \mid X=x\}$ using

$$
P\{\text { class } 2 \mid X=x\}=1-P\{\text { class } 1 \mid X=x\}
$$

\section{Let}

$$
\begin{aligned}
& f_{1}(x) \equiv \mathrm{P}\{\text { class } 1 \mid X=x\} \\
& f_{2}(x) \equiv \mathrm{P}\{\text { class } 2 \mid X=x\}
\end{aligned}
$$

Then the Overlap Ratio is then defined as:

$$
\text { overlap Ratio }=\frac{\text { Area under both } f_{1}(x) \text { and } f_{2}(x)}{\text { Area under } f_{1}(x)+\text { Area under } f_{2}(x)}
$$

The smaller the Overlap Ratio, the more easily the two classes can be discriminated.

\section{The SOGR-I and SOGR-IB classification algorithms}

\section{Overview}

The Self-Organizing Global Ranking (SOGR) algorithm [37] was inspired by Kohonen's Self-Organizing Map (SOM) algorithm [40]. In the SOM algorithm, each neuron has associated with it a topological neighborhood, and the algorithm is such that neighboring neurons in the topological space tend to arrange themselves over time into a grid in feature space that mimics the neighborhood structure in the topological space. The SOGR algorithm differs from the SOM algorithm by dropping the topological neighborhood and replacing it with the concept of a global neighborhood generated by ranking. We consider two variants of the SOGR algorithm:

- The first variant, SOGR-I [38,39], modifies the initialization scheme of SOGR.

- The second variant, SOGR-IB [38,39] ("B" stands for "Batch update"), removes the dependence on the order in which instances are presented by only updating the weights after each cycle, where a cycle involves presenting the entire training set to the network, one instance at a time. This variant also uses the modified initialization procedure described above.

Before we describe the above modifications in detail, we describe the SOGR algorithm itself.

\section{The SOGR classification algorithm}

We assume that $m$ neurons are used; each neuron $j$ has a weight vector $\vec{W}_{j}(t)$, where $t$ represents time. Let the initial position of neuron $j$ at time $t=0$ be $\vec{W}_{j}(0)$, and assume that the training set consists of instances $\left(\vec{x}_{i}, y_{i}\right)$, $i=1, \ldots, n$, where the $\vec{x}_{i}$ are feature vectors, and $y_{i}$ denotes the class of an instance.

1. Initialization: Choose initial positions $\vec{W}_{j}(0)$ in feature space for the $m$ neurons by assigning the neurons random positions in feature space.

2. Present the instances in the training set to the network, one at a time. As each instance is presented to the network, the time index $t$ is increased by 1 . For each instance $\left(\vec{x}_{i}, y_{i}\right)$ in the training set, the positions of one or more neurons are adjusted as follows:

- Identifying Winning Neurons: Find the $R$ closest neurons to the feature vector $\vec{x}_{i}$, that is, find the $R$ neurons with the smallest value of $\left\|\vec{x}_{i}-\vec{W}_{j}(t)\right\|$. These $R$ neurons constitute the "neighborhood" of the input vector. Let $\Gamma$ be the set of indices of the $R$ winning neurons. 
- Updating Weights: Adjust the positions of each of the $R$ winning neurons using the update rule

$$
\vec{W}_{j}(t+1)=\vec{W}_{j}(t)+\eta_{t}\left(\vec{x}_{i}-\vec{W}_{j}(t)\right)
$$

where $j \in \Gamma$ and $\eta_{t}$ is the learning rate. The learning rate is chosen to decrease with time in order to force convergence of the algorithm. In [37] it is suggested that the learning rate be decreased at an exponential rate, and that it should be smaller for larger neighborhood sizes $R$.

3. Assigning Classes to Neurons: Associated with each neuron $j$ is a count of the number of instances belonging to each class that are closer to neuron $j$ than any other neuron. This count is calculated as follows:

- For each neuron, initialize the counts to zero.

- For each instance $\left(\vec{x}_{i}, y_{i}\right)$ in the training set, find the closest neuron to the feature vector $\vec{x}_{i}$, that is, find the neuron with the index $j^{*}$, where

$$
j^{*}=\arg \min _{j}\left\|\vec{x}_{i}-\vec{W}_{j}(t)\right\|
$$

and increment the count in neuron $j^{*}$ corresponding to class $y_{i}$ by 1 .

- After all instances in the training set have been considered, each neuron is assigned to the class corresponding to the largest count for that neuron.

After the training process has been completed, a test instance can be classified by assigning it the class label of the nearest neuron.

\section{The SOGR-I classification algorithm}

The first variant, SOGR-I $[38,39]$, modifies the initialization scheme of SOGR. Specifically, assume that the feature space is $d$ dimensional, so that the feature vectors $\vec{x}_{i}$ belong to $\mathbb{R}^{d}$. For each feature $k$, we find the largest and smallest value of that feature over the entire training set, which are respectively $L_{k}$ and $U_{k}$ :

$$
\begin{aligned}
L_{k} & =\min _{i} x_{i k} \\
U_{k} & =\min _{i} x_{i k}
\end{aligned}
$$

where $x_{i k}$ is the $k^{\text {th }}$ element of the feature vector $\vec{x}_{i}$. Then the initial positions of the $m$ neurons are chosen as:

$$
W_{j k}(0)=L_{k}+\frac{j-1}{m-1}\left(U_{k}-L_{k}\right) \quad \begin{array}{r}
j=1, \ldots, m \\
k=1, \ldots, d
\end{array}
$$

Thus the $m$ neurons are evenly distributed along the line connecting $\left(L_{1}, L_{2}, \ldots L_{d}\right)$ to $\left(U_{1}, U_{2}, \ldots U_{d}\right)$. This approach has several advantages over other initialization methods:

- It guarantees that the neurons will be in some sense evenly distributed throughout the feature space. Random initialization, on the other hand, does not guarantee this. If one has a large feature space, say of 60 dimensions, and comparatively few neurons, say 50 , then with random initialization those neurons will with high probability not be evenly distributed throughout the feature space.

- Even a small number of neurons can be used to populate the feature space. If we consider an alternate initialization procedure in which one populates the feature space with a d-dimensional grid of neurons, and there are $q$ grid points along each feature space axis, then the total number of neurons required to populate this grid is $q^{d}$. For example, if $q=3$ and the feature space has 60 dimensions, then the number of neurons required is

$$
q^{d}=3^{60} \approx 4.239 \times 10^{28}
$$

which is clearly infeasible.

\section{The SOGR-IB classification algorithm}

The second variant, SOGR-IB $[38,39]$, addresses two problems with the original SOGR algorithm:

- The SOGR algorithm updates the weights after each new instance is presented to the network; as a result, the neuron trajectories can oscillate wildly.

- The SOGR algorithm specifies that the learning rate should be decreased during the course of training, for example at an exponential rate. The problem is that if the learning rate is decreased too rapidly, then the neurons may get stuck before they have reached their optimal positions.

SOGR-IB ("B" stands for "Batch update") addresses these problems in two ways:

- It uses a "batch update" strategy for updating the positions of the neurons in feature space. This eliminates the dependence of the results on the order in which instances are presented to the network, and also stabilizes the trajectories of the neurons.

- The batch update strategy allows the use of a fixed, but small, learning rate $\eta_{t^{\prime}}$ which eliminates the problem of 
the weights getting stuck because the learning rate $\eta_{t}$ was decreased too quickly.

The SOGR-IB algorithm is described below:

1. Initialization: Choose initial positions $\vec{W}_{j}(0)$ in feature space for the $m$ neurons using the SOGR-I initialization strategy. Set $t=0$.

2. Repeat the following until the "energy" defined by

$$
Q(t)=\frac{1}{2 n R} \sum_{\text {instances } i} \sum_{\text {neurons } j} m_{i j}\left\|\vec{x}_{i}-\vec{W}_{j}(t)\right\|^{2}
$$

does not reach a new minimum over a number of iterations through the training set, where $n$ is the number of training instances, $R$ is the number of neurons neighboring a given training instance that will be updated, and for each instance $\left(\vec{x}_{i}, y_{i}\right)$ in the training set, $m_{i j}=1$ for neurons $j$ that are one of the R closest neurons to the feature vector $\vec{x}_{i}$, and $m_{i j}=0$ for all other neurons $j$. After each pass through the training set, the time index $t$ is incremented by 1 .

(a) Let $\vec{Z}_{j}$ be the "accumulator" corresponding to neuron $j$. Initialize $\vec{Z}_{j}$ to 0 for all neurons $j$.

(b) Present the instances $\left(\vec{x}_{i}, y_{i}\right)$ in the training set to the network, one at a time. After each instance is presented, the "accumulators" are updated as follows:

- Identifying Winning Neurons: Find the $R$ closest neurons to the feature vector $\vec{x}_{i}$, that is, find the $R$ neurons with the smallest value of $\left\|\vec{x}_{i}-\vec{W}_{j}(t)\right\|$. These $R$ neurons constitute the "neighborhood" of the input vector. Let $\Gamma$ be the set of indices of the $R$ winning neurons.

- Updating Accumulators: Adjust the accumulators corresponding to each of the $R$ closest neurons using the update rule

$$
\vec{Z}_{j}=\vec{Z}_{j}+\frac{1}{n R} \eta_{t}\left(\vec{x}_{i}-\vec{W}(t)\right)
$$

where $j \in \Gamma$ and $\eta_{t}$ is the learning rate. (c). Updating Neurons: After all instances in the training set have been presented to the network, update the weights for each neuron $j$ using the rule:

$$
\vec{W}_{j}(t+1)=\vec{W}_{j}(t)+\vec{Z}_{j}
$$

where $n$ is the number of instances in the training set.

3. Assigning Classes to Neurons: Same as Step 3 in the SOGR algorithm above.

\section{Competing interests}

The authors declare that they have no competing interests.

\section{Authors' contributions}

JYY conceived of the project; MQY and JYY contributed ideas to the project; MQY designed the project; MQY performed the experiments and analyses, and wrote the manuscript; AKD, YPD and XH contributed suggestions.

\section{Acknowledgements}

We are indebted to Dr. Okan K. Ersoy of Purdue University, and Dr. Albert Overhauser of Purdue University for helpful discussions. Dr. Craig W. Codrington contributed ideas to the project, helped MQY perform the experiments and analyses, and helped MQY write the manuscript.

This article has been published as part of BMC Genomics Volume 9 Supplement I, 2008: The 2007 International Conference on Bioinformatics \& Computational Biology (BIOCOMP'07). The full contents of the supplement are available online at http://www.biomedcentral.com/|47|-2 |64/ 9? issue $=$ SI.

\section{References}

I. Chapman R, Sidrauski C, Walter P: Intracellular signaling from the endoplasmic reticulum to the nucleus. Annu Rev Cell Dev Biol 1998, 14:459-485.

2. Chen CP, Rost B: State-of-the-art in membrane protein prediction. Appl Bioinformatics 2002, I:2 I-35.

3. Soltys B], Gupta RS: Mitochondrial proteins at unexpected cellular locations: export of proteins from mitochondria from an evolutionary perspective. Int Rev Cytol 2000, 194: I33-196.

4. Gudermann T, Nurnberg B, Schultz G: Receptors and $G$ proteins as primary components of transmembrane signal transduction. Part I. G-protein-coupled receptors: structure and function. J Mol Med 1995, 73:51-63.

5. Heusser C, Jardieu P: Therapeutic potential of anti-IgE antibodies. Curr. Opin. Immunol 1997, 9:805-8I3.

6. Saragovi HU, Gehring K: Development of pharmacological agents for targeting neurotrophins and their receptors. Trends Pharmacol Sci 2000, 2 1:93-98.

7. Bagos PG, Liakopoulos TD, Hamodrakas SJ: Evaluation of methods for predicting the topology of $\beta$-barrel outer membrane proteins and a consensus prediction method. BMC Bioinformatics 2005, 6():7.

8. Moriyama EN, Kim J: Protein family classification with discriminant function analysis. In Genome Exploitation: Data Mining the Genome Edited by: Edited by Gustafson JP, Shoemaker R, Snape JW. New York: Springer; 2005.

9. Shimozono S, Shinohara A, Shinohara T, Miyano S, Kuhara S, Arikawa S: Knowledge Acquisition from Amino Acid Sequences by Machine Learning System BONSAI. Trans. Information Processing Society of Japan 1994, 35:2009-2018. [http://citeseer.ist.psu.edu/ 1081 19.html]

10. Casadio R, Fariselli P, Taroni C, Compiani M: A predictor of transmembrane $\alpha$-helix domains of proteins based on neural net- 
works. European Biophysics Journal 1996, 24(3):165-178. [http:// dx.doi.org//0.1007/BF00180274]

II. Dombi GW, Lawrence J: Analysis of protein transmembrane helical regions by a neural network. Protein Science 1994, 3(4):557-566. [http://www.proteinscience.org/cgi/content/abstract/ $3 / 4 / 557]$

12. Lohmann R, Schneider G, Behrens D, Wrede P: A neural network model for the prediction of membrane-spanning amino acid sequences. Protein Science 1994, 3(9):1597-160I. [http://www.pro teinscience.org/cgi/content/abstract/3/9//597]

13. Rost B, Casadio R, Fariselli P, Sander C: Transmembrane helices predicted at 95\% accuracy. Protein Science 1995, 4(3):52I-533. [http://www.proteinscience.org/cgi/content/abstract/4/3/52I]

14. Cai YD, Zhou GP, Chou KC: Support Vector Machines for Predicting Membrane Protein Types by Using Functional Domain Composition. Biophysical Journal 2003, 84(5):3257-3263. [http://www.biophysi.org/cgi/content/abstract/84/5/3257]

15. Lin HH, Han LY, Cai CZ, Ji ZL, Chen YZ: Prediction of transporter family from protein sequence by support vector machine approach. Proteins 2006, 62:2I8-23I. [http://dx.doi.org/ 10.1002/prot.20605]

16. Natt NK, Kaur H, Raghava GPS: Prediction of transmembrane regions of $\beta$-barrel proteins using $A N N$ - and SVM-based methods. Proteins 2004, 56: II-18.

17. Park KJ, Gromiha MM, Horton P, Suwa M: Discrimination of outer membrane proteins using support vector machines. Bioinformatics 2005, $21(23): 4223-4229$.

18. Yuan Z, Mattick JS, Teasdale RD: SVMtm: Support vector machines to predict transmembrane segments. Journal of Computational Chemistry 2004, 25(5):632-636. [http://dx.doi.org/ 10.1002/jcc.104II]

19. Sonnhammer ELL, von Heijne G, Krogh A: A hidden Markov model for predicting transmembrane helices in protein sequences. In Proceedings of the 6th International Conference on Intelligent Systems for Molecular Biology (ISMB) Menlo Park, CA: AAAI Press; 1998:175-182. sonnhammer98hidden.html]

20. Viklund $H$, Elofsson $A$ : Best $\alpha$-helical transmembrane protein topology predictions are achieved using hidden Markov models and evolutionary information. Protein Science 2004, 13(7): 1908-19|7. [http://www.proteinscience.org/cgi/content/ abstract//3/7//908]

21. Radivojac P, Obradovic Z, Smith DK, Zhu G, Vucetic S, Brown CJ, Lawson JD, Dunker AK: Protein flexibility and intrinsic disorder. Protein Science 2004, 13:7|-80.

22. Peng K, Vucetic S, Radivojac P, Brown CJ, Dunker AK, Obradovic Z: Optimizing Long Intrinsic Disorder Predictors with Protein Evolutionary Information. J Bioinform Comput Biol 2005, 3:35-60.

23. lakoucheva LM, Radivojac P, Brown CJ, O'Connor TR, Sikes JG, Obradovic $Z$, Dunker AK: The importance of intrinsic disorder for protein phosphorylation. Nucleic Acids Res 2004, 32(3): 1037-1049.

24. Romero P, Dunker AK: Intelligent Data Analysis for Protein Disorder Prediction. Artificial Intelligence Review 2000:14.

25. Dunker AK, Obradovic Z: The protein trinity-linking function and disorder. Nature Biotechnology 200I, I 9(9):805-806.

26. Kyte J, Doolittle R: A simple method for displaying the hydropathic character of a protein. J. Mol. Biol 1982, I 57:105-132.

27. Eisenberg D, Schwarz E, Komaromy M, Wall R: Analysis of membrane and surface protein sequences with the hydrophobic moment plot. Journal of Molecular Biology 1984, 1 79: I 25-I 42.

28. Engelman DM, Steitz TA, Goldman A: Identifying nonpolar transbilayer helices in amino acid sequences of membrane proteins. Annu. Rev. Biophys. Biophys. Chem 1986, 15:321-353.

29. Liu LP, Deber CM: Guidelines for membrane protein engineering derived from de novo designed model peptides. Biopolymers (Peptide Science) 1998, 5(47):41-62.

30. Grantham R: Amino acid difference formula to help explain protein evolution. Science 1974, I 85(4154):862-864.

31. Zimmerman JM, Eliezer N, Simha R: The characterization of amino acid sequences in proteins by statistical methods. J. Theor. Biol | 968,2 I (2): 170-20|.

32. Ortolani F, Raspanti M, Marchini M: Correlations between amino acid hydrophobicity scales and stain exclusion capacity of type I collagen fibrils. J. Electron Microscopy 1994, 43:32-8.
33. Dwyer DS: Electronic properties of amino acid side chains: quantum mechanics calculation of substituent effects. $B M C$ Chemical Biology 2005, 5(2): I-II.

34. Liu LP, Deber CM: Uncoupling Hydrophobicity and Helicity in Transmembrane Segments. J. Biol. Chem 1998, 273(37):23645-23648. [http://www.jbc.org/cgi/content/abstract/273/ 37/23645]

35. Quinlan JR: C4.5: Programs for Machine Learning. San Francisco: Morgan Kaufmann; 1993.

36. Joachims T: Making large-Scale SVM Learning Practical. In Advances in Kernel Methods - Support Vector Learning Edited by: Edited by Schölkopf B, Burges C, Smola A. MIT Press; 1999.

37. Saglam MI, Ersoy O, Erer I: Self-Organizing Global Ranking Algorithm and its Applications. In Intelligent Engineering Systems Through Artificial Neural Networks, Volume 14 2004:893-898.

38. Yang MQX, Yang JY, Codrington CW: Identification of Transmembrane Proteins Using Variants of the Self-Organizing Feature Map Algorithm. In Knowledge Discovery in Bioinformatics: Techniques, Methods and Applications Edited by: Edited by Pan Y, Hu X. John Wiley \& Sons; 2006.

39. Yang MQX: Predicting Protein Structure and Function Using Machine Learning Methods. In PhD thesis Purdue University, West Lafayette, Indiana; 2005.

40. Kohonen T: Self-organizing formation of topologically correct feature maps. Biological Cybernetics 1982, 43:59-69.

Publish with Bio Med Central and every scientist can read your work free of charge

"BioMed Central will be the most significant development for disseminating the results of biomedical research in our lifetime. "

Sir Paul Nurse, Cancer Research UK

Your research papers will be:

- available free of charge to the entire biomedical community

- peer reviewed and published immediately upon acceptance

- cited in PubMed and archived on PubMed Central

- yours - you keep the copyright
BioMedcentral 